\title{
Contents, Vol. 123, 1952
}

\section{Index}

Algan, B., vide Ch. Thomas.

Amsler, M., Kystes de Гiris 241

Discussion: Goldmann. Amsler, M., und L, Hoffmann-Egg, Eine Iochverdächtíge Stelle in der Netzhaut 269

Diskussion: Goldmann. Arató, L, and M. Arató, Cholinesterase Activity in the Serum and

Aqueous in

Glaucoma $\quad 374$

Arató, M., vide /. Arató.

Bagóczky, L., Improved Technique in Operations for Pterygium 60

- A New Instrument for Peeling off Residual Portions of the Apex of aPterygium

- $\quad$ Marginofixator. A New Instrument for Holding the Lid Margin in Position 124

Barth, J., Dystrophia myotonica 338

Bischler, V., Le Pseudo-Phénomène de Graefe congenital 322

Blum, J. D., Aphakie unilatérale et verres de contact 284

Böhringer, H. R., Sekundärglaukom mit Gefäßneubildungen auf der Iris . . 211 Bornschein, H., vide G. Schubert.

Bouman, M. A., and G. van den Brink, On Night Myopia 100

Van den Brink, G., vide M. A. Bouman.

Bürki, E., i)ber den klinischen Wert der Röntgenaufnahme des Canalis oplicus 243

Diskussion: Thiel, Huber, A. Bruckner, Rintelen. Schlußwort: Bürki. Cavka, V., Über die

Augensymptome bei Encephalitis chronica in Verbindung

mit Gehirnkalzifikationen 78

Cordier, J., vide Ch. Thomas.

Cuendet, J.-F., E. Rosselet et C. Meneghini, Observations cliniques avec des nou-

veaux collyres isotoniques et tamponnés $\quad 334$

Cuendet, J. F., vide R. Dufour.

Dufour, R., Indications particulières des verres de contact: kératite neuroparalytique, albinisme 290

Discussion à Blum et Dufour: Amsler, Dufour, Goldmann, Verrey.

Conclusion: Dufour. Dufour, R., et J. F. Cuendet, Appreciation quantitative des perturbations des functions visuelles. (A propos d'un cas de traumatisme du nerf optique) . 249Dufour, M., vide U. P. Portmann.Franceschetti, A., Le strabisme concomitant aigu 219

Discussion: Dufour, Amsler. Franceschetti, A., et J. H. Rickli, Névrite rétrobulbaire après intoxication aiguë

aux hydrocarbures chlorés 255

Goldmann, H., Die Wírkungsweise der Iridektomie beim chronisch kongestiven

Glaukom 202

- $\quad$ Ein neues Spaltlampenfluorometer 277 
Diskussion: Huber. Schlußwort: Goldmann.

De Haas, E. B., Deux complications oculaires rares de la maladie de Besnier-Bceck chez un même sujet. Kératoconjonctivite sèche et calcification de lacornée et de la conjunctive 65 Haselmann, G., K. Pulfrich und H. Haselmann, Zur Behandlung von Epithelschäden der Cornea 357

Haselmann, H., vide G. Haselmann.

Hoffmann-Egg, L, Sympathische Ophthalmie nach Iridencleisis mit ausgedehnter epiretinaler Zellanhäufung über den Netzhautgefäßen 207

Diskussion: Moeschlin.

- vide M. Amsler.

Huber, A., Einseitige Stauungspapille Pseudoneuritis und Pseudopapillenödem 261

Diskussion: A. Bruckner, Goldmann.

Huber, 0., Ophthalmologisches aus dem Glarnerland

198

Jean-Sédan et S. Sédan-Bauby, A propos de deux cas d'hypertension oculaire

après ovario-hystérectomie 150

Kaleff, R., Meine vereinfachte Modifikation der Dacryocystorhinostomia externa

ohne Nähte 157

Klimková-Deutschová, E., and J. Velický, Stargardt's Degeneration of the Retina

with Neurological and Endocrine Complications 162

Kretschmar, S., A propos de la fausse correspondance rétinienne .... 343

Discussion: Goldmann.

- La coordimétrie au périmètre de Goldmann _ . 348

Lakatos, I., Arachnoiditis optico-chiasmatica46

De Leonibus, F., Akkommodative Richtungsänderung der Augenachse ... 15 Van Mane $\pi$, J. G., «The Plank»; a Method for a Better Objective Examination

of Infants 51

Du Marchie Sarvaas, G. J., An Instrument for Use in Squint Operations . . 122

Meneghini, C, vide J.-F. Cuendet.

Messikommer, W., Akuter Keratokonus bei einem Kinde 326

Diskussion: Rintelen, Böck.Mortelmans, L., Forme familiale de la dystrophie cornéenne de

Fuchs ... 88Moser, R., Gefahren der Cortisontherapie $\quad 313$

Diskussion: Franceschetti, Goldmann, Saubermann.

Mailer, P., Cortison. Indikation und Anwendung 310

Netıenschwander, M., Homöopathie in der Augenheilkunde 316

Pascal, J. I., Lens Replacement of Accommodative Activity 394

Pau, H., Permeabilitätskatarakt und intrakapsuläre Staroperation .... 40

- Über scheinbare Abdichtung bzw. Durchlässigkeitserhöhung von Linsendurch

Nichtelektrolyte $\quad 144$

Paviëic, Z., Beitrag zur operativen Verschmälerung der Lidspalte .... 57 Pitte $\tau$, J., und J. Svejda, Über den Einfluß der Röntgenstrahlen auf die Ent-

stehung von Mißbildungen der menschlichen Frucht 386

Portmann, U. P., et M. Dufou $\tau$, Indications et contre-indications données par

Torthoptique dans le traitement du strabisme convergent concomitant . . 294

Discussion: Rintelen, Meyer, Kretzschmar. Conclusion: Portmann. Prijot, E., vide R. Weekers.

Pulfrich, K., vide G. Haselmann. Remky, E., Zum Bewegungssehen und zur

Pendelpeitschenschmitzentäuschung 
(Whiplash Illusion) 168

Rickli, J. H., vide A. Franceschetti.

Rintelen, F., Zur Frage der Blutungsprophylaxe bei bulbuseröffnenden Opera-

tionen 228

Diskussion: Thiel, Huber, Amsler, Dufour, R. Bruckner, Böhringer.

Schlußwort: Rintelen.

- Die Farbenphotographie im klinischen Unterricht 320

Schlußwort: Rintelen.

Rosselet, E., vide J.-F. Cuendet.

Rosselet, E., vide E. B. Streifj.

Sakic, D., Un cas de Dysgenesis Mesodermalis Corneae et Iridis (Rieger) . . 31

Saubermann, G., Die Bedeutung des Applikationsmodus bei antibiotischer

Therapie 303

Diskussion: Dufour. Schlußwort: Saubermann. Schubert, G., und H. Bornschein, Beitrag zur

Analyse des menschlichen Elektro-

retinogramms 396

Sédan-Bauby, S., vide Jean-Sédan.

Streiff, E. B., et E. Rosselet, Plaies perforantes, foyers infectieux, ophtalmie

sympathique 235

Discussion: Verrey, Dufour. Svejda, J., vide/. Pitter.

Taboriski, J., The Late Bodies of Trachoma ' $\quad .383$

Thiel, R., Zur Pathogenese des primären Glaukoms 195

Diskussion: Rintelen, Goldmann. Schlußwort: Thiel. Thomas, Ch., J. Cordier et B. Algan, La

lymphoréticulose bénigne oculo-gan-

glionnaire 129

Velický, J., vide E. Klimková-Deutschová.

Verrey, F., Kritische Studien über Mißerfolge bei Netzhautoperationen (erste

Mitteilung) 271

Diskussion: A. Bruckner. Schlußwort: Verrey. Vrabec, Fr, Sur la question de Гendothélium de

la surface antérieure de $\Gamma$ iris

humain 20

Weekers, R., et E. Prijot, Mesure de la resistance à Гécoulement de Thumeur

aqueuse au moyen du tonomètre électronique.

IrePartie: Résultats chez le sujet normal

1

2e Partie: Résultats chez les sujets atteints de glaucome chronique non in-

flammatoire 114

3e Partie: Résultats chez les sujets glaucomateux opérés par la diathermie

rétrociliaire thermométrique 365

Weidmann, W., Vorführung eines neuen Adaptometers 355

Diskussion: Goldmann.

Wettler, H., t)ber Augensymptome bei Gargoylismus 338

Witmer, R., Elektrophorese des Kammerwassers 280

Diskussion: Amsler, Goldmann, Verrey. Witmer, R., Erfahrungen mit dem Sero-Test auf

Tuberkulose nach Middlebrook

und Dubos 329

Diskussion: Böck. 
- $\quad$ Uveitis chronica bei Bronchiektasien 352

Diskussion: Gruber, Witmer.

- $\quad$ Eine spezielle Form rezidivierender Chorioiditis. (Hierzu Tafel I) 353Zollinger, R., Klinische Untersuchungen über Gefäßneubildungen auf der Iris . 216

Diskussion: Goldmann, Rintelen. Schlußwort: Zollinger.

GESELLSCHAFTSBERICHTE - SOCIETY TRANSACTIONS - SOC1ÉTËS

The Netherlands Ophthalmological Society. 121st Meeting, held on 9th and 10th

December, 1950, in the Netherlands Eye Hospital, Utrecht 180

Schweizerische Ophthalmologische Gesellschaft. 44. Generalversammlung inGlarus, 28. bis 30.

September 1951. - Société Suisse d'Ophtalmologie. 44eAssemblée générale à Glaris, 28-30

septembre $1950 \quad 193$

BUCHBESPRECHUNGEN - BOOK REVIEWS - LIVRES NOUVEAUX

$63,125,190,413$

V. Varia 64

128

416

AПe Rechte vorbehalten Cliches: Aberegg-Steíner \& Cie. AG.

Bern. Buchdruckerei Fñedrich Reinhardt AG.

Basel 